18-454 Illingworth

5-3-18v1

Ulipristal Acetate for Treatment of Symptomatic Uterine Leiomyomas: A Randomized Controlled Trial

We read with interest the randomized controlled trial evaluating ulipristal acetate for the treatment of symptomatic uterine leiomyomas by Simon et al. ${ }^{1}$ The authors should be congratulated for undertaking a multicenter randomized trial evaluating the efficacy of ulipristal acetate for the treatment of symptomatic uterine leiomyomas in such a diverse population.

The authors described a commendable objective to "evaluate the efficacy and tolerability of ulipristal acetate," however, we wonder if an emphasis on safety would have been beneficial in light of concerns recently expressed by the European Medicines Agency. ${ }^{2}$

The European Medicines Agency has recommended no new women should be started on ulipristal acetate after reports of serious liver injury, including liver failure leading to transplantation. They advise all women currently taking ulipristal acetate should have a liver function test at least once every 4 weeks during treatment and repeated 2-4 weeks after stopping treatment. If the test is abnormal, treatment should be stopped, and the women closely monitored. ${ }^{2}$ It would be useful for the authors to clarify their clinical practice recommendations considering the recent European Medicines Agency advice.

In the near future, regulatory decisions will be informed by systematic reviews of published evidence. Would the authors consider publishing any data related to deranged liver function, hepatitis, liver failure, or liver transplant collected as part of their trial? As any future assumption that they were absent is likely to be correct but may not be secure. ${ }^{3}$ Moving forward we would advocate for the development of a minimum data set to improve safety reporting in future research, permitting a more balanced assessment by considering the trade-off between the benefits and harms of ulipristal acetate. ${ }^{4}$ 
18-454 Illingworth

5-3-18v1

2

\section{Financial Disclosure}

The authors did not report any potential conflicts of interest.

Mr Benjamin JG Illingworth

Martin Hirsch MRCOG ${ }^{2}$

James M. N. Duffy MBChB MRes ${ }^{3}$

${ }^{1}$ University College London Medical School, London, WC1E 6BT, United Kingdom

${ }^{2}$ Queen Mary, University of London, London, E1 2AB, United Kingdom.

${ }^{3}$ Balliol College, University of Oxford, Oxford, OX1 3BJ, United Kingdom.

\section{Correspondence to:}

Dr James M. N. Duffy MBChB MRes BSc (Hons) PG Cert HCL

Balliol College, University of Oxford, Oxford, OX1 3BJ, United Kingdom.

+447949066806

james.duffy@balliol.ox.ac.uk

@jamesmnduffy

\section{Word count: 273 words}

\section{References}

${ }^{1}$ Simon JA, Catherino W, Segars JH, Blakesley RE, Chan AP, Sniukiene V, AI-Hendy A.

Ulipristal Acetate for Treatment of Symptomatic Uterine Leiomyomas: A Randomized

Controlled Trial. Obstet Gynecol 2018;131(3):431-439.

${ }^{2}$ Web site: European Medicines Agency. Esyma. Available at:

www.ema.europa.eu/ema/index.jsp?curl=pages/medicines/human/referrals/Esmya/human_r eferral_prac_000070.jsp\&mid=WC0b01ac05805c516f\&source=homeMedSearch\&category= human 
18-454 Illingworth

$5-3-18 \mathrm{v} 1$

${ }^{3}$ Duffy JMN, Hirsch M, Pealing L, Showell M, Khan KS, Ziebland S, McManus RJ.

60 Inadequate safety reporting in pre-eclampsia trials: a systematic evaluation. BJOG; in press.

$61{ }^{4}$ Duffy JMN, Rolph R, Gale C, Hirsch M, Khan KS, Ziebland S, McManus R. Core outcome

62 sets in women's and newborn health: a systematic review. BJOG 2017; 124(10):1481-1489. 